\title{
Characterization of Acoustic Effects in Ferroelectric Thin-Films for Microwave Components
}

Giere, A.; Schafranek, Robert; Zheng, Yuliang et al.

(2008)

DOI (TUprints):

License:

Publication type:

Division:

Original source: https://doi.org/10.25534/tuprints-00013399

only the rights of use according to UrhG

Article

11 Department of Materials and Earth Sciences

18 Department of Electrical Engineering and Information Technology https://tuprints.ulb.tu-darmstadt.de/13399 


\title{
Characterization of Acoustic Effects in Ferroelectric Thin-Films for Microwave Components
}

\author{
By A. Giere, R. Schafranek, Y. Zheng, H. Maune, M. Sazegar, R. Jakoby, and A. Klein
}

\begin{abstract}
In this paper we present the impact of acoustic resonances excited by electrostriction in BST thin-films on the $\mathrm{Q}$-factor of a varactor in the $\mathrm{GHz}$ range. By changing the process-parameter substrate to target distance while sputtering the thin-films, we observe a change in the acoustic resonance frequencies. To characterize the material properties which determine the acoustic resonance frequencies a model based on a 1D acoustic transmission line is used. The extracted material properties results in a proportional dependency to the changed process parameter. For verification of the acoustic transmission line model 3D simulations have been performed which show good agreement to the line model for lower resonance frequencies, where the difference at higher frequencies becomes significant due to additional higher order modes.
\end{abstract}

Index Terms - Acoustic effects, modeling, thin-film, ferroelectric, characterization, barium strontium titanate

\section{Introduction}

Tunable devices are key components in future microwave applications like mobile communications. Their utilization promises an increase in functionality and in parallel a decrease of the number of components and their size. Typical applications for tunable devices are phase-shifters, tunable filters and impedance matching networks. They can be realized in the microwave range by using the ferroelectric (FE) material barium strontium titanate (BST) $[1,2,3,4]$ which offers in contrary to the already established semiconductor technology the opportunity of passive tuning [5]. For building up components based on BST thin-film as well as thick-film techniques are commonly used. Where BST thick-films are in general structured planar, thin-films are used to realize stakked Metal-Insulator-Metal (MIM) structures. The dielectric layers in both techniques can be tuned by an external electric field, which reduces the permittivity passively and affected the capacitance value directly.

For the efficient use of such tunable capacitances (varactors) in the microwave region, a quite high Q-factor is required which is affected by the dielectric losses, the limited conductivity of metal electrodes and especially in MIM structures by acoustic resonances. In contrary to the dielectric and ohmic losses, the losses induced by acoustic resonances are exited by electrostriction only when an external electric field is applied [6]. For typical thin-film structures, where the film thickness is in the order of 200-400 nm and the electrode thicknesses are below $1 \mu \mathrm{m}$, several acoustic resonances are exciting in different small frequency bands below $10 \mathrm{GHz}$. For the design of thin-film based tunable MIM capacitors the acoustic properties of the whole component has to be known, therefore the composition of the several stacked layers has to be analyzed and optimized with the goal of shifting the narrow frequency bands where acoustic resonances are exited out of the preferred working frequency bands.

Up to now the modeling of the acoustic resonances in such FE varactors is quite uncommon, not only due to the limited number of software tools which enable the computation of the varactors response from acoustic resonances in the microwave domain, but also due to the precise knowledge of the acoustic and piezoelectric properties. For modeling the acoustic response of thin-film varactors [6] and [7] presented an acoustic transmission line model based on a 1D model of the stacked layers. Due to considering only material properties, coupling and responses aligned in the direction of the normal surface vector this method is limited in mapping the real 3D structure. software tools like ANSYS are currently able to simulate the ferroelectric varactor impedance in 3D considering acoustic resonances in the microwave domain. But nevertheless the extraction of the required material properties from measurement results can effectively only be done with the simple 1D transmission line model due to it's processing speed advantage.

In this paper we characterize the acoustic properties of the stacked layer of five BST thin-films where the material properties are varied by changing the target to substrate distance during sputter deposition. Finally the used 1D transmission line model is compared with $3 \mathrm{D}$ multi-physic simulations.

\section{Preparation and characterization of ferroelectric thin-film based microwave components}

BST thin-films of $300 \mathrm{~nm}$ thickness were deposited via RF magnetron sputtering on $\mathrm{Si}(300 \mu \mathrm{m})(100) / \mathrm{SiO}_{2}(300 \mathrm{~nm}) /$ $\mathrm{TiO}_{2}(20 \mathrm{~nm}) / \mathrm{Pt}(150 \mathrm{~nm})(111)$ substrates. The substrate to target distance $d$ was varied between $5 \mathrm{~cm}$ and $10 \mathrm{~cm}$ while the sputtering power density of $2.5 \mathrm{~W} / \mathrm{cm}^{2}$, the substrate temperature of $650^{\circ} \mathrm{C}$, the working pressure of $5 \mathrm{~Pa}$ and the $\mathrm{Ar} / \mathrm{O}_{2}$ ratio of 99/1 were kept constant. On top of the sputtered BST thin-films Pt top electrodes of $400 \mathrm{~nm}$ thickness were structured using lift-off lithography to realize the MIM-varactors.

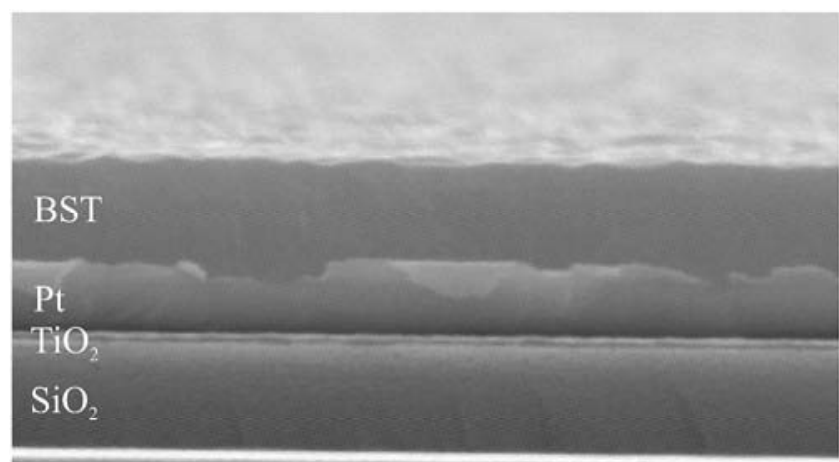

$\mathrm{Si}$ $500 \mathrm{~nm}$

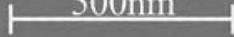

Fig. 1: High resolution scanning electron microscope image of the crosssection of the $\mathrm{Si}(100) / \mathrm{SiO}_{2} / \mathrm{TiO}_{2} / \mathrm{Pt}(111) / \mathrm{BST}$ layer stack deposited at a target to substrate distance of $d=8.8 \mathrm{~cm}$. 
Table 1: Overview of the BST thin-film properties as the lateral grain size $D$, the root-mean-square roughness $R_{\text {rms }}$ measured by AFM, the $\mathrm{Ba} / \mathrm{Sr}$ ratio, the $\mathrm{Ti}$ excess $y=\mathrm{Ti} /(\mathrm{Ba}+\mathrm{Sr})-1$ deduced from RBS measurements and deposition rate $R$ in function of the substrate to target distance $d$ during sputter deposition.

\begin{tabular}{|l|c|c|c|c|c|}
\hline$d[\mathrm{~cm}]$ & $D[\mathrm{~nm}]$ & $R_{\mathrm{rms}}[\mathrm{nm}]$ & $\mathrm{Ba} / \mathrm{Sr}$ & $y$ & $R[\mathrm{~nm} / \mathrm{min}]$ \\
\hline 5 & 190 & 1.7 & $0.57 / 0.43$ & 0.26 & 7 \\
\hline 6.3 & 190 & 1.8 & $0.56 / 0.44$ & 0.27 & 4.5 \\
\hline 7.5 & 213 & 2.5 & $0.57 / 0.43$ & 0.09 & 2.4 \\
\hline 8.8 & 135 & 3.8 & $0.56 / 0.44$ & 0.07 & 1.4 \\
\hline 10 & 108 & 4.5 & $0.56 / 0.44$ & 0.04 & 0.85 \\
\hline
\end{tabular}

Table 2: Acoustic resonance frequencies and associated Q-factors of substrate with a target to substrate distance of $d=10 \mathrm{~cm}$.

\begin{tabular}{|c|c|c|c|c|c|}
\hline $\begin{array}{c}d \\
{[\mathrm{~cm}]}\end{array}$ & $\begin{array}{c}f_{1}[\mathrm{GHz}] / \\
Q_{1}\end{array}$ & $\begin{array}{c}f_{2}[\mathrm{GHz}] / \\
Q_{2}\end{array}$ & $\begin{array}{c}f_{3}[\mathrm{GHz}] / \\
Q_{3}\end{array}$ & $\begin{array}{c}f_{4}[\mathrm{GHz}] / \\
Q_{4}\end{array}$ & $\begin{array}{c}f_{5}[\mathrm{GHz}] / \\
Q_{5}\end{array}$ \\
\hline 5 & $2.48 / 8.4$ & $5.32 / 3.0$ & $9.23 / 20.0$ & $11.5 / 13.1$ & $14.68 / 6.5$ \\
\hline 6.3 & $2.42 / 8.5$ & $5.37 / 2.7$ & $9.07 / 17.8$ & $11.3 / 11.8$ & $14.60 / 8.3$ \\
\hline 7.5 & $2.40 / 4.1$ & $5.31 / 1.4$ & $8.93 / 14.4$ & $11.30 / 7.9$ & $14.53 / 5.3$ \\
\hline 8.8 & $2.38 / 6.2$ & $5.35 / 2.4$ & $8.79 / 15.5$ & $11.24 / 9.2$ & $14.40 / 7.1$ \\
\hline 10 & $2.37 / 5.2$ & $5.15 / 1.9$ & $8.70 / 13.1$ & $10.96 / 9.9$ & $14.20 / 5.8$ \\
\hline
\end{tabular}

A cross section of the $\mathrm{Si} / \mathrm{SiO}_{2} / \mathrm{TiO}_{2} / \mathrm{Pt} / \mathrm{BST}$ layer stack without the processed top electrodes made by high resolution scanning electron microscopy (HRSEM) is shown in Figure 1.

The morphology of the BST film surface was studied using a ThermoMicroscopes Autoprobe CP research atomic force microscope (AFM) in contact mode. An AFM image is shown in Figure 2.

As shown in Table 1 the deposition rate $R$ decreases with increasing target to substrate distance during the sputter deposition from $7 \mathrm{~nm} / \mathrm{min}$ for $d=5 \mathrm{~cm}$ to $0.85 \mathrm{~nm} / \mathrm{min}$ for $d=10 \mathrm{~cm}$, leading to decrease of lateral grain size from

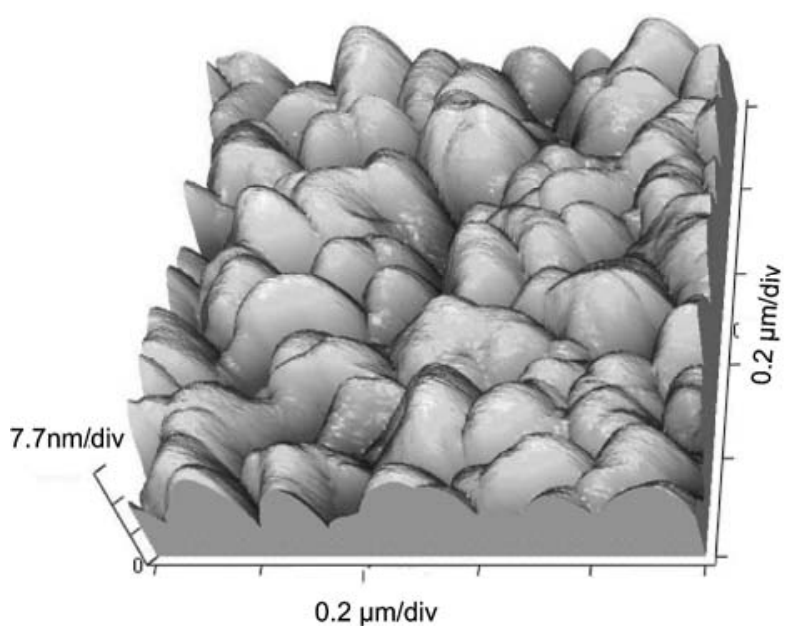

Fig. 2: Atomic force microscope image of the BST thin-film surface of the sample shown in Figure 1.

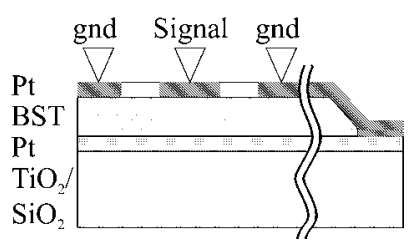

a)

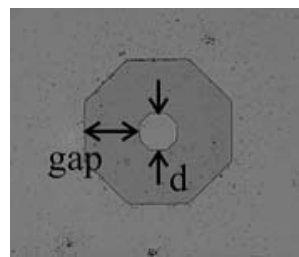

b)
Fig. 3: a) Schematic cross-section of the BST thin-film capacitor with the GSG-Probe for the RF measurement on top. b) Top view on the realized varactor with $\mathrm{d}=26 \mu \mathrm{m}$ and gap $=41 \mu \mathrm{m}$.

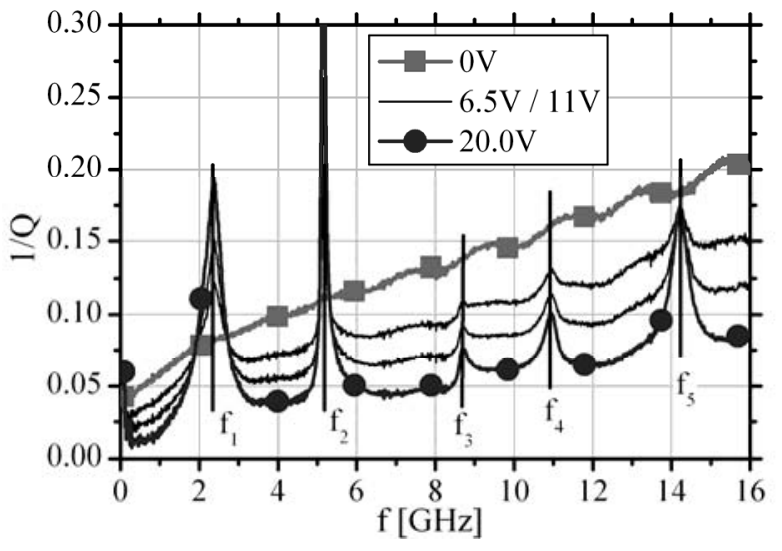

Fig 4: The rising acoustic resonances of the BST thin-film can be seen when applying a tuning voltage from $U=0 \mathrm{~V}$ to $U=20 \mathrm{~V}$ to the varactor prepared at a target to substrate distance of $d=10 \mathrm{~cm}$.

$D \sim 200 \mathrm{~nm}$ to $D \sim 110 \mathrm{~nm}$ and an increase of the root-meansquare roughness from $R_{\mathrm{rms}}=1.7 \mathrm{~nm}$ to $R_{\mathrm{rms}}=4.5 \mathrm{~nm}$.

This behavior can be explained by larger nucleation sites as a result of the reduced deposition rate at larger $d[8]$. The BST thin-film composition was analyzed by Rutherford Backscattering Spectrometry (RBS). The Ba/Sr ratio and Ti excess $y=\mathrm{Ti} /(\mathrm{Ba}+\mathrm{Sr})-1$ are presented in Table 1 . While the $\mathrm{Ba} / \mathrm{Sr}$ ratio remains rather constant a strong increase of the Ti excess from $y=0.04(d=10 \mathrm{~cm})$ to $y=0.26(d=5 \mathrm{~cm})$ can be observed with decreased substrate to target distance.

\section{Microwave response of BST thin-film components}

The realized BST varactors are measured at a controlled temperature of $22^{\circ} \mathrm{C}$. To contact the varactor, Ground-SignalGround (GSG) probes are used as shown in the cross-section in Figure $3 \mathrm{a}$ within an on-wafer measurement setup.

The tunable capacitor $C$ is formed by an octagon shaped pad (see Fig 3b) connected by the signal contact of the GSGprobes and the Pt-layer below the BST film. The lower electrode is connected for the RF signal trough the large capacitance from the top gnd-layer to the bottom layer which acts on the microwave signal as a short circuit. For being able to apply a bias voltage $U$ to the capacitance, the bottom layer and the top gnd-layer are hot-wired at an edge of the substrate. The dielectric properties of the five deposited thin-films were first of all presented in [9]. They show a distinct influence of the sputter target to substrate distance to the BST thin-films properties. Their permittivity, their tunability and their lossfactor all measured at $1 \mathrm{MHz}$ vary between $\varepsilon_{\mathrm{r}}=200 \ldots 500$, $\tau(50 \mathrm{~V} / \mu \mathrm{m})=26 \% \ldots .65 \%$ and $\tan \delta_{\max }=0.02 \ldots 0.0032$ respectively when the distance changes between $d=10 \mathrm{~cm}$ and $d=5 \mathrm{~cm}$. These effects can be described by the increased Ti excess in the films shown by $y$ in Table 1 .

In Figure 4 the 1/Q plot illustrates the acoustic resonances rising up by tuning voltage. The Q-factor is measured in the frequency range of $40 \mathrm{MHz}$ up to $16 \mathrm{GHz}$ to be able to monitor five acoustic resonances at $f_{1}$ to $f_{5}$ in the substrate processed with $d=10 \mathrm{~cm}$.

For further material characterization the acoustic resonance frequencies and the minimum Q-factor at these resonances have been extracted from the measurements. These characteristics are summarized in Table 2 . The tuning voltage is limited to $\mathrm{U}=20 \mathrm{~V}$ which results in an electric tuning field strength of $66.7 \mathrm{~V} / \mu \mathrm{m}$ within the ferroelectric thin-film.

The variation of the resonance frequencies as well as the dependency on the minimum Q-factor at the resonance shows a dependency to the varied process parameter. It can be as- 
sumed that the changing morphology of the film results in a change of the films stiffness. The change of the minimum Qfactor is related to the changing surface roughness of the films.

It should be mentioned that the observed resonance frequencies $f_{n}$ are not matched with the simple theory (eq. 1) of unloaded acoustic resonators [10] of a length $l_{p}$ which corresponds to the BST film thickness and its acoustic velocity $c_{p}$

$f_{n}=\frac{n \cdot c_{p}}{l_{p}} \quad n \in \mathbf{N}$

Therefore in the following section a transmission line model for the acoustic resonator is presented, taking the influence of the used metal and dielectric layers adjacent to the BST layer into account.

\section{Modeling the acoustic impedance using the transmission line model}

The method used for modeling the impact of acoustic resonances excited by electrostriction in a stacked layer capacitor is based on the acoustic transmission line model presented in [6] and [7]. The stacked layer structure of the realized BST thin-film varactors is shown in Fig. 5a. This representation neglects different material properties at the interface between two layers and assumes constant homogeneous material properties in each layer.

The acoustic properties including the layer thickness of these layers are summarized in Table 3 .

The calculation of the impedance $Z$ of the varactors based on the normalized acoustic load impedance $z_{t}$ and $z_{b}$ on the top and bottom of the piezoelectric layer respectively is shown in eq. 2 and illustrated in Fig. 5 b by a simplified impedance model.

$Z=\frac{1}{j \omega C}\left(1-K^{2} \frac{\tan \phi}{\phi} \cdot \frac{\left(z_{t}+z_{b}\right) \cos ^{2} \phi+j \sin 2 \phi}{\left(z_{t}+z_{b}\right) \cos 2 \phi+j\left(z_{t} z_{b}+1\right) \sin 2 \phi}\right)$

$z_{t}=Z_{t} / Z_{p} ; z_{b}=Z_{b} / Z_{p} ; \phi=\left(\omega l_{p}\right) /\left(2 c_{p}\right)$

The tuning voltage dependent coupling between the applied electric field and the piezoelectric response of the thin-film is considered with the field dependent coupling coefficient $K^{2}$. It can be calculated from eq. 3 based on the effective piezoelectric coefficient $d_{33}^{* 2}(E)$, the elastic compliance $s_{33}$ and the tunable permittivity $\varepsilon_{33}(E)$ of the film.

$K^{2}(E)=\frac{d_{33}^{* 2}(E)}{s_{33} \varepsilon_{33}(E) \varepsilon_{0}+d_{33}^{* 2}(E)}$

Whereby the coupling coefficient and further the impedance of the capacitor only consider the material properties, coup-

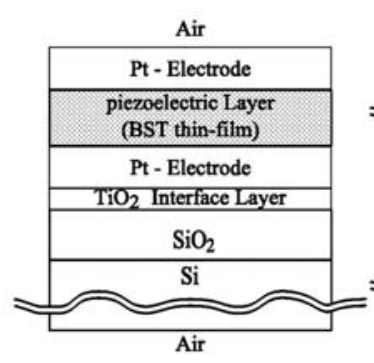

a)

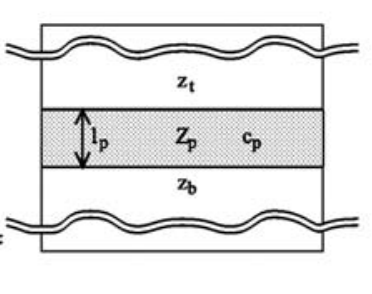

b)
Fig. 5: a) Stacked layer structure of the BST thin-film varactor used for modeling by the acoustic transmission line model. b) Simplified model for the acoustic impedances of the BST thin-film and at its interfaces.
Table 3: Acoustic properties of the used dielectric and metal layers used for the realization of BST thin-film varactor.

\begin{tabular}{|c|c|c|c|c|c|}
\hline Layer & $I_{\text {Layer }}[\mathrm{nm}]$ & $C_{\text {Layer }}[\mathrm{m} / \mathrm{s}]$ & $Z_{\text {Layer }}\left[\mathrm{kg} / \mathrm{m}^{2} \mathrm{~s}\right]$ & $Q_{\text {Layer }}$ & Reference \\
\hline Air & $\infty$ & 360 & 400 & & \\
\hline Pt-Top & 400 & 4230 & $57.6 \times 10^{6}$ & 150 & {$[6]$} \\
\hline $\mathrm{BST}$ & $I_{p}$ & $c_{p}$ & $Z_{p}$ & $Q_{p}$ & \\
\hline Pt-Bottom & 150 & 4230 & $57.6 \times 10^{6}$ & 150 & {$[6]$} \\
\hline $\mathrm{TiO}_{2}$ & 20 & 5200 & $22 \times 10^{6}$ & 500 & {$[11]$} \\
\hline $\mathrm{SiO}_{2}$ & 300 & 5100 & $12.55 \times 10^{6}$ & 500 & {$[6]$} \\
\hline $\mathrm{Si}$ & $300 \times 10^{3}$ & 8433 & $19.7 \times 10^{6}$ & 100 & {$[6]$} \\
\hline Air & $\infty$ & 360 & 400 & & \\
\hline
\end{tabular}

ling and responses in the direction of the applied electric tuning field and the microwave field denoted by the index 33 . The acoustic impedance at each boundary can be calculated from one of the adjacent layers. It is calculated by a transmission line model, given in eq. 4 .

$Z_{T}=\frac{Z_{b}+Z_{l} \tan \left(\omega l / c_{l}\right)}{Z_{l}+j Z_{b} \tan \left(\omega l / c_{l}\right)}$

The iterative calculation of the impedance on the lower boundary of the BST film starts at the air interface by setting the acoustic impedance $Z_{b}$ to the acoustic impedance of air This impedance is transformed by the acoustic line formed by the Si substrate having the impedance $Z_{l}$, a length $l$ and the velocity $c_{l}$ into the transformed impedance $Z_{T}$ at the interface between $\mathrm{Si}$ and $\mathrm{SiO}_{2}$. This impedance is transformed in a further step by the next layer to the impedance at the next interface since this interface attached the BST film. The same procedure is used to calculate the impedance of the layers on top of the BST film.

\section{Extraction of the acoustic material properties using the acoustic transmission line model.}

Due to the simple analytic equations the acoustic transmission line model is suited for an extraction of the unknown material properties of the BST film (acoustic impedance and acoustic velocity) from the measurements.

For reduction of the unknown parameters a constant coupling coefficient $K^{2}$ of 0.12 is used for modeling. This simplification is confirmed by the observed independency of the coupling coefficient on the observed acoustic resonance frequencies as shown in Fig. 4. This behavior is analytical proofed by eq. 2 where a change of $K^{2}$ has no effect to the response of the equation which denotes the acoustic resonances.

For the extraction a parameter sweeping technique is used which varies all unknown parameters independently of each other in a predicted range. For each parameter combination the frequency dependent impedance of the varactor is calculated and the frequencies of the arising acoustic resonances are monitored. By minimizing the error between the calculated resonance frequencies and the measured ones we are extract the material properties.

The extracted result of the BST thin-film properties shows a mismatch between the measured resonance and modelled frequencies even for the extraction results with the lowest error. This behavior is determined by different process technologies in literature for the materials. So the material properties (see Table 3) are varying in an unknown range. In addition the determination of the layer thickness of the $\mathrm{Pt}$ electrodes and of the BST film is only possible within a tolerance of $\sim 2 \%$. To consider all these variations the number of unknowns for the parameter sweep has to be increased from 
Table 4: Acoustic properties of five BST thin-films extracted with the transmission line model.

\begin{tabular}{|c|c|c|c|}
\hline$d[\mathrm{~cm}]$ & $I_{p}[\mathrm{~nm}]$ & $c_{p}[\mathrm{~m} / \mathrm{s}]$ & $Z_{p}\left[10^{6} \mathrm{~kg} / \mathrm{m}^{2} \mathrm{~s}\right]$ \\
\hline 5 & 298 & 10345 & 48.4 \\
\hline 6.3 & 341 & 11046 & 35.8 \\
\hline 7.5 & 323 & 11088 & 36.7 \\
\hline 8.8 & 299 & 11631 & 34.1 \\
\hline 10 & 312 & 11984 & 36.2 \\
\hline
\end{tabular}

Table 5: Comparison of acoustic resonance frequencies between measurement $f_{\mathrm{IM}}$ and FEM method $f_{\mathrm{FEM}}$

\begin{tabular}{|c|c|c|c|c|c|}
\hline $\begin{array}{c}d \\
{[\mathrm{~cm}]}\end{array}$ & $f_{1 M} / f_{1 F E M}$ & $f_{2 M} / f_{2 F E M}$ & $f_{3 M} / f_{3 F E M}$ & $f_{4 M} / f_{4 F E M}$ & $f_{5 M} / f_{5 F E M}$ \\
\hline 5 & $\begin{array}{c}2.48 / \\
2.44\end{array}$ & $\begin{array}{l}5.32 / \\
5.64\end{array}$ & $\begin{array}{c}9.23 / \\
9.11\end{array}$ & $11.5 / 11.1$ & $\begin{array}{c}14.68 / \\
15.39\end{array}$ \\
\hline 6.3 & $\begin{array}{c}2.42 / \\
2.24\end{array}$ & $\begin{array}{l}5.37 / \\
5.49\end{array}$ & $\begin{array}{c}9.07 / 1 \\
9.26\end{array}$ & $11.3 / 10.9$ & 14.6 / 15.1 \\
\hline 7.5 & $2.4 / 2.41$ & $5.31 / 5.9$ & $\begin{array}{l}8.93 / \\
9.95\end{array}$ & $11.3 / 11.7$ & $14.53 / 13.5$ \\
\hline 8.8 & $\begin{array}{l}2.38 / \\
2.48\end{array}$ & $\begin{array}{l}5.35 / \\
6.17\end{array}$ & 8.79 / n.a. & $11.24 / 12$ & 14.4 / 13.6 \\
\hline 10 & $\begin{array}{c}2.37 / \\
2.51\end{array}$ & $\begin{array}{l}5.15 / \\
6.17\end{array}$ & 8.7 / n.a. & $\begin{array}{c}10.96 / \\
11.9\end{array}$ & $14.2 / 13.5$ \\
\hline
\end{tabular}

two to 15 with additional sweeping the values by $\pm 2 \%$ compared to the literature values.

The extraction results for the BST thin-film acoustic properties are summarized in Table 4.

They show a dependency of the acoustic velocity on the varied process parameter and the associated change in the thin-film morphology. The much higher variation in BST film thickness can be explained by an imprecise film thickness determination using UV-Ellipsometry.

\section{Acoustic 3D-modelling of ferroelectric varactors}

The extracted acoustic properties from the transmission line model are verified by a tridimensional finite-element-method (FEM). The commercial FEM solver of ANSYS Multiphysics has been used for coupled field analysis. The model contains the necessary degrees of freedom, i.e. voltage and displacement. The interaction between the coupled electric and stress fields are represented by the piezoelectric matrix. With the fulfilled condition of linear systems it is able to solve the mechanical displacement under the applied high frequency voltage. Since only the elastic coefficient matrix and the density are accepted instead of acoustic velocity and impedance, necessary interpretations are carried out.

The capacitors are modeled with their finite dimensions, stack of materials and approximate boundary conditions. Hence, the resonant frequencies, not only the fundamental modes, but also the parasitic modes can be predicted at a low error level by the modal analysis [11]. The longitudinal element size is set to be a quarter of acoustic wavelength at the fundamental frequency, which reduces the complexity and may however lead to error at high order harmonics. With the material properties in Table 3 and 4 the resonant frequencies are comparable with those in Table 2. Their deviation is up to $6 \%$ in fundamental resonant frequencies, and $11 \%$ in higher order harmonics.

It is known that in stratified structures the longitudinal strain will couple to the transverse direction [12]. When the BST film thickness is thin compared to the diameters of contact pads and to the overall size of the continuous layer the infinite extent of the BST film can be assumed, which suppress transverse resonance modes. However, transverse propagation is still visible as shown in Fig. 6. It leads to reduced ac- curacy in the extraction of piezoelectric coupling coefficients, which desires further investigation.

\section{Conclusion and Outlook}

In this paper we presented the impact of acoustic resonances excited by electrostriction in BST thin-films on the Q-factor of a varactor in the $\mathrm{GHz}$ range. A parameter study of different thin-films with varied sputter target to substrate distance has been carried out. The material properties have been determined with a $1 \mathrm{D}$ acoustic transmission line model. The extracted acoustic impedance and velocity show a proportional dependency on the Ti excess as well as on the changed morphology which are related to the variation of the target to substrate distance during sputtering. The 1D transmission line model has been verified with a full 3D finite-element-method solver for multi-physics simulations. For low orders of acoustic resonances the $1 \mathrm{D}$ and $3 \mathrm{D}$ calculations show a quite good agreement. For higher order resonance frequencies the 1D transmission line model shows a larger deviation from the 3D calculations, due to a coupling from longitudinal strain into the transverse direction which can not be considered in the 1D model.

At microwave frequencies, the parasitic losses from metal electrodes and acoustic resonances are dominant. For the further optimization of the microwave properties the capacitor layout and electrode thickness have to be improved to reduce these parasitics. Using the presented results a further device optimization for BST based microwave components can be performed in the future.

\section{References}

[1] A. Kozyrev, et.al.,"Ferroelectric films: nonlinear properties and applications in microwave devices", Microwave Symposium Digest, 1998 IEEE MTT-S International, 1998

[2] A. Tombak, J. Maria, F. Ayguavives, Z. Jin, G. Stauf, A. Kingon and A. Mortazawi, "Voltage-controlled RF filters employing thin-film barium-strontium-titanate tunable capacitors", IEEE Transactions on Microwave Theory and Techniques, 2003, 51, pp. 462-467

[3] P. Scheele, F. Goelden, A. Giere, S. Mueller and R. Jakoby, "Continuously tunable impedance matching network using ferroelectric varactors", Microwave Symposium Digest, 2005 IEEE MTT-S International, 2005

[4] M. Schmidt, E. Lourandakis, A. Leidl, S. Seitz and R. Weigel, "A Comparison of Tunable Ferroelectric Pi and T-Matching Networks", Proc. 37th European Microwave Conf. 2007

[5] A. Tagantsev, V. Sherman, K. Astafiev, J. Venkatesh and N. Setter, "Ferroelectric Materials for Microwave Tunable Applications", Journal of Electroceramics, vol. 11, pp-5-66, 2003.

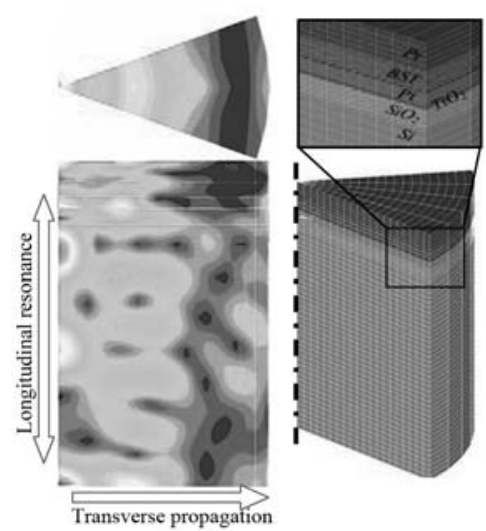

Fig. 6: Displacement contour of longitudinal resonance and transverse propagation on stratified structure due to layer stretch. A $10^{\circ}$ section is simulated with periodic boundary condition. (hotter color denotes larger displacement) 
Frequenz $62(2008)$
[6] S. Gevorgian, A. Vorobiev and T. Lewin, ,dc field and temperature dependent acoustic resonances in parallel-plate capacitors based on SrTiO3 and Ba0.25Sr0.75TiO3 films - Experiment and modeling“, Journal of Applied Physics, vol. 99, pp. 124112-11, 2006

[7] P. Turalchuk, I. Vendik, O. Vendik and J. Berge, ,Modelling of Tuneable Acoustic Resonators based on BSTO Films with Induced Piezoelectric Effect", Proceedings of the 37th European Microwave Conference, 2007

[8] M. Ohring, Materials Science of Thin Films. Deposition and Structure. New York: Academic Press, 2001

[9] R. Schafranek, A. Giere, A.G. Balog, T. Enz, Y. Zheng, P. Scheele, R. Jakoby, and A. Klein, ,Infuence of sputter deposition parameters on the properties of tunable barium strontium titanate thin-films for microwave applications", submitted

[10] M. Schmidt, ,Abstimmbare Anpassnetzwerke auf Basis ferroelektrischer Varaktoren für Mobilfunkanwendungen“, Technische Fakultät, Universität Erlangen-Nürnberg, 2007

[11] K. Nakamura, H. Kobayashi, H. Kanbara, „Evaluation of acoustic properties of thin films using piezoelectricovertone thickness-mode resonators“, IEEE Ultrasonics Symposium, 2000, Vol. 1, pp. $593-$ 597

[11] D.Boucher, M. Lagier, C. Maerfeld, Computation of the Vibrational Modes for Piezoelectric Array Transducers using a Mixed Finite Element-Perturbation Method, IEEE, Trans. on Sonic. and Ultrasonics. Vol. SU-28, No. 5, pp. 318-330, Sep 1981

[12] A. Reinhardt, S. Ballandras and V. Laude, Simulation of Transverse Effects in FBAR Devices, 2005
This work was supported in part by the DFG Research Training Group 1037 "Tunable integrated components in microwave technology and optics".

Andre Giere, Yuliang Zheng, Holger Maune, Mohsen Sazegar, Rolf Jakoby

Microwave Engineering

Technische Universität Darmstadt

Merckstrasse 25

64289 Darmstadt

Germany

Fax: +49 6151/16-4322

E-mail: giere@hf.tu-darmstadt.de

Robert Schafranek and Andreas Klein

Institute of Materials Science

Surface Science Division

Technische Universität Darmstadt

Petersenstrasse 23

64287 Darmstadt

Germany

Fax: +49 6151166308

(Received on February 28, 2008) (Revised on March 19, 2008)

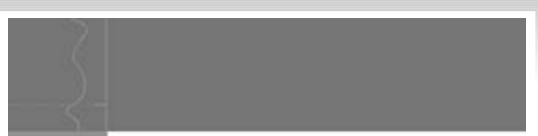

Journal of Optical Communications

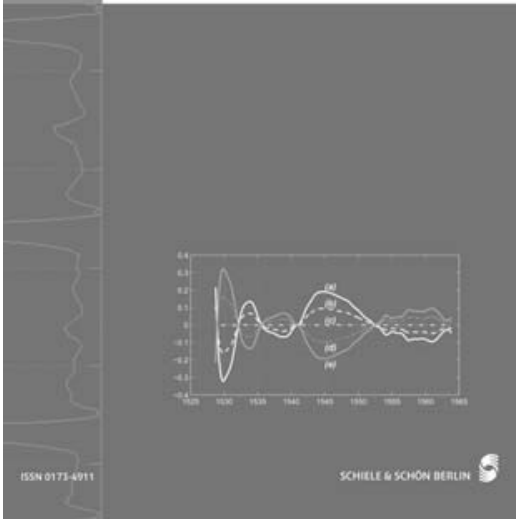

\section{Excellency in international research}

The renowned international publication covering all fields of optical communications. The bimonthly Journal of Optical Communications provides the scientific community all over the world with high-quality information.

Publication language is English.

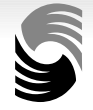

SCHIELE \&SCHÖN

Fachverlag

Schiele \& Schön GmbH Markgrafenstr. 11

10969 Berlin

Tel.: +49 (30) $253752-0$

Fax: +49 (30) $253752-99$ E-Mail

service@schiele-schoen.de

Internet

www.schiele-schoen.de

\section{Order now!}

\section{Annual Subscription "Journal of Optical Communications" (free of Shipping costs): Germany $€ 501$; USA and outside Europe $€ 521.40$; within Europe $€ 510.60$ (plus VAT). Annual subscriptions are payable for a year in advance. Cancellations must reach the publishers by November 15 .}

Orders can be placed at any bookshop or with the publishers.
Name/Company

Address

Country zipcode/City

Telephone

E-Mail 